\title{
Double non-disjunction in maternal meiosis II giving rise to a fetus with $48, \mathrm{XXX},+21$
}

\author{
Vicki M Park, Ralph R Bravo, Lee P Shulman
}

\begin{abstract}
We describe a prenatally detected case of double trisomy involving chromosome 21 and the $X$ chromosome $(48, X X X,+21)$ along with determination of the segregation errors responsible for the double aneuploidy. The patient was ascertained as a result of an abnormal maternal serum analyte screen showing an increased risk for fetal Down's syndrome. Following determination of the abnormal karyotype, pregnancy termination was elected. Microsatellite polymorphisms and cytogenetic heteromorphisms were used to determine that both aneuploidies arose as a result of non-disjunction in maternal meiosis II. These results support hypotheses that a segregation defect at a cellular level may cause non-disjunction involving more than one chromosome.
\end{abstract}

(f Med Genet 1995;32:650-653)

Although non-disjunction is the most common cause of chromosomal abnormalities, the presence of two numerical abnormalities in a single conceptus is rarely observed. A number of such cases have been reported, ${ }^{1-7}$ but the mechanisms by which double aneuploidies arise have not been well studied. We report a fetus with both trisomy 21 and trisomy $\mathrm{X}$ in which both aneuploidies arose by maternal non-disjunction in meiosis II.

Traditionally malsegregation of chromosomes has been studied using cytogenetic heteromorphisms. ${ }^{89}$ This type of analysis is relatively subjective, as visual differentiation of subtle polymorphisms may be problematical. The usefulness of such analyses is limited to those human chromosomes that frequently exhibit heterochromatic polymorphisms, including chromosomes 1, 9, 16, and the acrocentric chromosomes. On the other hand, since the polymorphic heterochromatin is localised to pericentromeric regions, cytogenetic heteromorphisms lend themselves to the analysis of a centromere driven event such as segregation. These studies have assumed an absence of recombination between the heteromorphism and the adjacent centromere. Available evidence from meiotic studies supports this assumption ${ }^{10}$ but is limited to observations of normal male meioses.

More recently, polymorphic DNA markers have been applied to the study of chromosome segregation..$^{11-14}$ Since DNA markers are highly polymorphic and very abundant, it is possible to identify informative loci in almost all families. Furthermore, since DNA polymorphisms are distributed throughout the genome, the segregation of any chromosome can be evaluated. The primary limitation of the molecular approach is that commonly used markers are located some distance away from the centromere, and integration of the centromere into the genetic map has not been achieved for most chromosomes. Therefore, as with cytogenetic heteromorphisms, recombination events occurring between the polymorphism and the centromere cannot be detected, potentially leading to incorrect assessment of the stage of meiotic error. Studies comparing results from cytogenetic heteromorphisms and from molecular markers have noted significant discrepancies between the two systems with respect to stage of meiotic error. ${ }^{131516}$ Such discrepancies could be explained by increased recombination in pericentromeric regions. ${ }^{1718}$ However, there is no question that, when used judiciously, molecular approaches greatly improve our ability to study segregation. This is especially true of the type of case reported here, where segregation analyses of two chromosomes are required.

\section{Case report}

The parents were healthy and unrelated 19 year olds, and the mother had had one previous pregnancy that resulted in the delivery of a normal, healthy girl. In the current pregnancy, amniocentesis was performed at 17.5 weeks' gestation following a "positive" serum analyte screen for fetal Down's syndrome (adjusted risk of 1/120). Ultrasonography at the time of amniocentesis showed nuchal thickening $(10 \mathrm{~mm})$, a large ventricular septal defect, and a two vessel cord. The karyotype showed trisomy 21 and trisomy $\mathrm{X}$ in all 20 cells examined (fig 1a). The parents elected pregnancy termination, which was performed at 19 weeks' gestation. Routine confirmation of the fetal karyotype could not be accomplished owing to gross microbial contamination of all cultures initiated from products of conception. Therefore, interphase FISH (fluorescence in situ hybridisation) with probes for chromosomes $\mathrm{X}$ and 21 was used to confirm the presence of both trisomies in fetal tissue. The karyotypes of both parents were normal.

\section{Methods}

Cytogenetic analyses of GTG banded chromosomes from amniotic fluid and peripheral blood were performed according to standard procedures. Interphase FISH was performed according to a touch prep protocol from Imagenetics, Inc (Framingham, MA). Briefly, a freshly cut surface of fetal tissue was pressed 
a

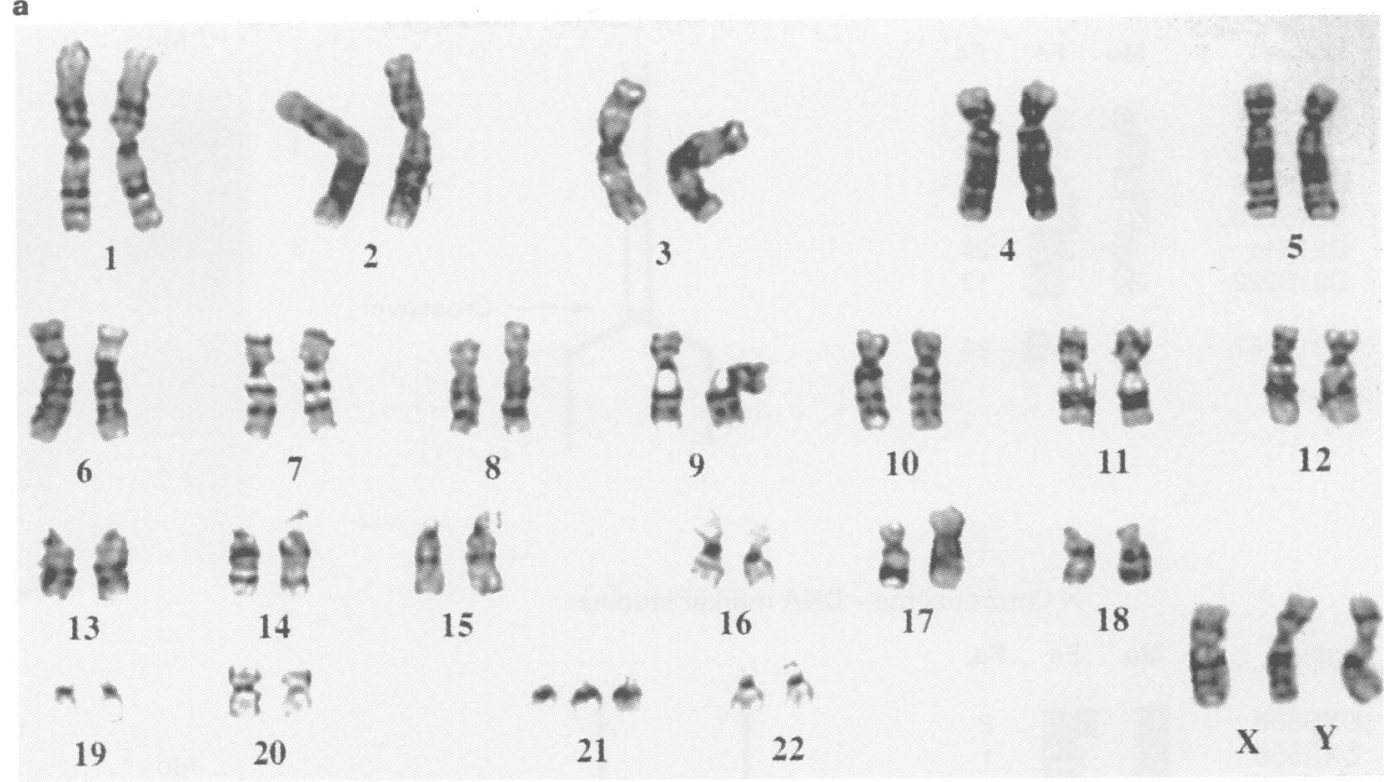

b

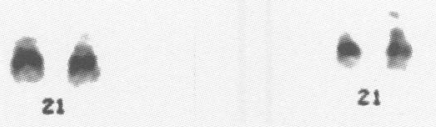

Figure 1 (a) Fetal karyotype from amniotic fluid, 48,XXX, +21. (b) Partial karyotypes of chromosome 21 from the mother (left) and the father (right).

lightly against a clean microscope slide in order to deposit cells onto the slide. Following fixation, slides were processed for FISH. The probes used to confirm the double trisomy were a centromeric $\mathrm{X}$ chromosome probe (DXZ1) and a cosmid probe from the long arm of chromosome 21 (21q22.3); hybridisation conditions were those recommended by the supplier (Oncor, Inc, Gaithersburg, MD). Using the DXZ1 probe, $98 \%$ of fetal cells exhibited three hybridisation signals, while control slides containing normal diploid cells yielded three signals in only $0.5 \%$ of cells. Hybridisation of the $21 \mathrm{q}$ probe was less efficient overall, but showed the presence of trisomy 21 cells in the fetal specimen. Approximately $10 \%$ of fetal cells exhibited three signals, while no such cells were observed in control slides.

DNA was extracted from either peripheral blood or minced fetal tissue by cell lysis, proteinase $\mathrm{K}$ digestion, and phenol/chloroform extraction. ${ }^{19}$ Oligonucleotide primer sets specific for microsatellite polymorphisms were obtained from Research Genetics (Huntsville, AL). Genotyping was performed by polymerase chain reaction (PCR) using a "hot start" protocol. AmpliWax beads were used according to specifications provided by the supplier (Perkin Elmer/Applied Biosystems). Briefly, each $100 \mu \mathrm{l}$ reaction contained $10 \mathrm{mmol} / 1$ Tris $-\mathrm{HCl}$, $\mathrm{pH} 8.3,50 \mathrm{mmol} / 1 \mathrm{KCl}, 2.5 \mathrm{mmol} / 1 \mathrm{MgCl}_{2}$, $0.2 \mathrm{mmol} / 1$ each of dATP, dTTP, dCTP, and dGTP, $4 \mu \mathrm{Ci}^{32} \mathrm{P}$-dCTP, $0.5 \mathrm{mmol} / 1$ primers, 3 units Taq DNA polymerase, and 50 ng genomic DNA. Before initiation of the reaction, beads were used to segregate enzyme and DNA from the remainder of the reagents in each tube. PCR conditions were 30 cycles of $94^{\circ}$ (10 seconds), $55^{\circ}$ (30 seconds), and $72^{\circ}$ (30 seconds). The initial cycle was preceded by five minutes at $94^{\circ}$, and the final cycle was followed by five minutes at $72^{\circ}$. Reaction products were run on $6 \%$ polyacrylamide/urea gels followed by autoradiography. For each locus examined, observed alleles were assigned numbers based on relative order of migration in the gel.

\section{Results}

Microsatellite analysis was used to investigate the parental origin of the extra chromosomes observed in the fetus, and both the extra chromosome 21 and the extra $\mathrm{X}$ chromosome were found to be of maternal origin (fig 2). Evidence of recombination was detected on both chromosomes, since reduction to homozygosity was observed at some alleles while maternal heterozygosities were maintained at others. At the more distal loci (D21S167 on chromosome 21 and MAOA, DXS556, and DXS538 on the X chromosome), the fetus had inherited three different alleles, two of which were of maternal origin. Since the fetus had inherited two different maternal alleles at these loci, neither aneuploidy could have arisen through postzygotic mitotic error. All other loci examined confirm a maternal origin of the extra chromosomes. Based on visual inspection of comparative band intensities within lanes containing fetal DNA, the fetus inherited a single maternal allele at each proximal locus (D21S215, D21S258, D21S236, D21S225, D21S11, and D21S222 on chromosome 21 and ALAS2 and DXS981 
Chromosome 21 - DNA marker studies

$\begin{array}{llll}\text { Locus } & \text { Mo } & \text { Fe } & \text { Fa } \\ & & & \\ \text { D21S215 } & 13 & 233 & 12 \\ \text { D21S258 } & 23 & 122 & 13 \\ \text { D21S236 } & 24 & 344 & 13 \\ \text { D21S225 } & 23 & 225 & 12 \\ \text { D21S11 } & 13 & 233 & 24 \\ \text { D21S222 } & 23 & 133 & 12 \\ \text { D21S167 } & 14 & 134 & 23\end{array}$

\section{$X$ Chromosome - DNA marker studies}

$\begin{array}{lccc}\text { Locus } & \text { Mo } & \text { Fe } & \text { Fa } \\ \text { DXS538 } & 13 & 123 & 2 \\ \text { DXS556 } & 23 & 123 & 1 \\ \text { MAOA } & 13 & 123 & 2 \\ \text { ALAS2 } & 23 & 22 & 1 \\ \text { DXS981 } & 12 & 113 & 3\end{array}$

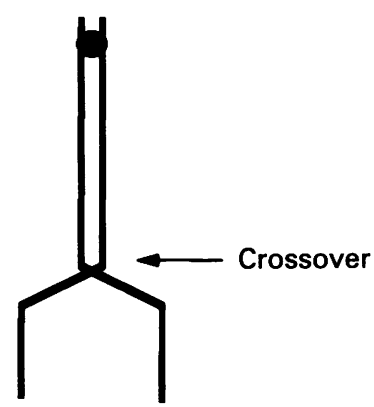

3

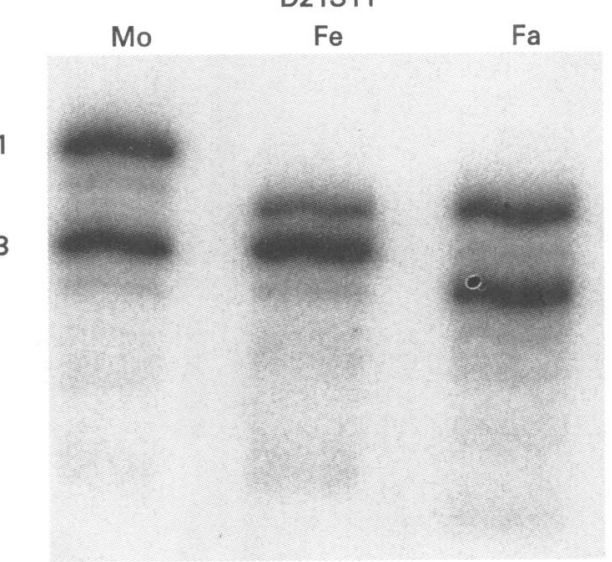

Fetus $=233$

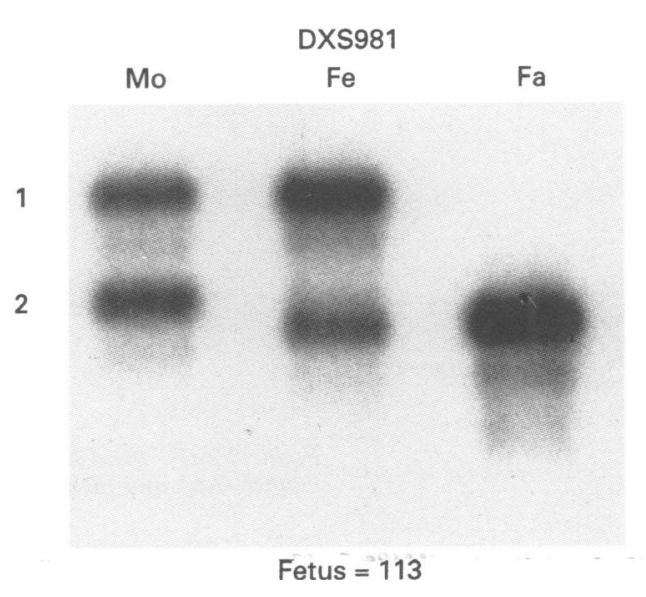

Figure 3 Two loci showing maternal meiosis II nondisjunction of chromosome 21 (D21S11) and the $X$ chromosome (DXS981). Alleles observed in the mother (Mo), fetus ( $\mathrm{Fe}$ ), and father ( $\mathrm{Fa}$ ) are numbered sequentially.

For the $\mathrm{X}$ chromosome, five informative loci were identified (fig 2). Relative map order of these loci is according to Schlessinger et $a l^{22}$ and Brown et al. ${ }^{23}$ The single informative long arm locus was DXS981, and the fetus inherited two copies of the maternal allele 1 (figs 2 and 3). Likewise, the most proximal short arm locus, ALAS2, exhibited reduction to homozygosity of maternal allele 2 . These two markers, flanking the centromere, indicate that the $\mathrm{X}$ chromosome non-disjunction also occurred in maternal meiosis II. For the remaining three informative markers, located distal to ALAS2 on $\mathrm{Xp}$, heterozygosity of the maternally derived alleles was maintained in the fetus. This indicates the occurrence of a crossover between ALAS2 and MAOA. metaphase preparations were evaluated for the presence of cytogenetic heteromorphisms involving chromosome 21 . In the mother, there was a clear difference in the lengths of the stalk regions of the two copies of chromosome 21 (fig 1b). This difference was not observed in metaphase preparations from the fetus, where all three short arm segments appeared to be approximately equal in length (fig 1a). This suggests that the fetus received a double dose of one maternal chromosome, rather than one copy of each homologue. As with the molecular data, this observation is consistent with the occurrence of a meiosis II non-disjunction.

\section{Discussion} double trisomy, in which both non-disjunction events occurred in maternal meiosis II. By examination of microsatellite polymorphisms on $\mathrm{Xp}, \mathrm{Xq}$, and $21 \mathrm{q}$, as well as cytogenetic heteromorphisms on $21 \mathrm{p}$, we obtained consistent data from the pericentromeric region of each chromosome arm. The observed $21 \mathrm{p}$ heteromorphism would not have been in-
We describe a case of prenatally detected 
formative on its own but, in combination with the molecular data, was useful in evaluating the stage of the meiotic error. As mentioned previously, recombination occurring between the centromere and the most proximal marker can lead to incorrect assessment of the stage of meiotic error. The use of pericentromeric loci on each chromosome arm minimises this possibility, since only a missed double crossover would lead to an incorrect conclusion.

Double trisomies are rarely observed, presumably because double non-disjunctions are rare events, associated with inevitable lethality in most cases. Reported cases of multiple aneuploidies have described liveborns exhibiting two viable aneuploidies (most often aneuploidy of the sex chromosomes combined with either trisomy 13,18 , or 21$) \cdot{ }^{1-7}$ The case described here also involved a complement of this type (48, $\mathrm{XXX},+21)$. Other cases of multiple aneuploidy involving at least one non-viable trisomy have been observed in spontaneous abortions. ${ }^{2425}$

Although few cases of multiple aneuploidy have been investigated (see below), several mechanisms may be considered by which they might arise. All require a minimum of two errors in cell division. Random non-disjunction in both gametes could lead to the formation of a doubly aneuploid zygote. Alternatively, a single global defect might cause multiple nondisjunctions in the formation of a single gamete.

Available experimental evidence favours the second possibility as the predominant cause of multiple aneuploidy. DNA polymorphisms have been used to investigate parental origins of both aneuploid chromosomes in three cases of spontaneous abortion, and in each case both non-disjunctions occurred in a single parent. ${ }^{25}$ In these three cases, stage of meiotic error was not determined for both aneuploidies. Parental origin of the extra chromosomes has also been investigated in cases of sex chromosome polysomy. In reported cases, each aneuploidy results from segregation errors in a single parent. ${ }^{26-28}$ For example, in reported cases of pentasomy $X(49, X X X X X)$, there are four $X$ chromosomes of maternal origin as a result of successive $\mathrm{X}$ chromosome non-disjunction in maternal meioses I and II. ${ }^{262829}$ It has been postulated that the successive errors are related, in that perturbed recombination may affect both meiotic segregations. ${ }^{182526}$ In the case reported here, the two non-disjunction events are related by virtue of occurring not only in the same parent, but in the same cell division. The presence of a general cellular defect, such as impaired spindle function or improper signalling of sister chromatid segregation, might account for this type of event. .0-33 $^{3}$

Study of additional examples of multiple aneuploidy are needed to determine the nature of the errors in such cases. Evaluation of exceptional instances of segregation failure undoubtedly will be useful in improving our understanding of the general mechanisms of non-disjunction.

We acknowledge the expert technical assistance of Ms Jody Lerner, and we thank Mr Russell Dodd for preparation of the figures.
1 Ford $\mathrm{CE}$, Jones $\mathrm{KW}$, Miller OJ, et al. The chromosomes in a patient showing both mongolism and Klinefelter syndrome. Lancet 1959;i:709-10.

2 Hecht F, Nievaard JE, Duncanson N, et al. Double aneuploidy: the frequency of XXY in males with Down's syndrome. Am $\mathcal{f}$ Hum Genet 1969;21:352-9.

3 Mailhes JB, Moore CM, Gershanik JJ. A case of double trisomy in a liveborn infant: $48, \mathrm{XXY},+13$. Clin Gene 1977;11:147-50.

4 Townsend G. Dentition of a 48,XYY, +21 male. Hum Genet 1982;61:267-8.

5 Therman E, Susman M. Human chromosomes: structure, behavior, and effects. 3rd ed. New York: Springer-Verlag, 1993:198-9.

6 Jaruratanasirikul $\mathrm{S}$, Jinorose $\mathrm{U}$. An infant with double trisomy $(48, \mathrm{XXX},+18)$. Am $₹$ Med Genet 1994;49:207-10.

7 Tsukahara M, Fukuda M, Furukawa S. Double trisomy (48,XXX, +18). Am ₹ Med Genet 1994;52:244.

8 Hassold TJ, Jacobs PA. Trisomy in man. Annu Rev Genet 1984;18:69-97.

9 Bricarelli FD, Pierluigi M, Landucci M, et al. Parental age and the origin of trisomy 21: a study of 302 families. Hum Genet 1989;82:20-6.

10 Laurie DA, Hulten MA. Further studies on bivalent chiasma frequency in human males with normal karyotypes. Ann Hum Genet 1985;49:189-201.

11 Antonarakis SE, The Down Syndrome Collaborative Group. Parental origin of the extra chromosome in trisomy 21 as indicated by analysis of DNA polymorphisms. $N$ Engl $f$ indicated by analysis $1991 ; 324: 872-6$.

12 Sherman SL, Takaesu N, Freeman SB, et al. Trisomy 21 association between reduced recombination and nondisjunction. Am $₹$ Hum Genet 1991;49:608-20.

13 Petersen MB, Frantzen $M$, Antonarakis SE, et al. Comparative study of microsatellite and cytogenetic markers for detecting the origin of the nondisjoined chromosome 21 in Down syndrome. Am $\mathcal{f}$ Hum Genet 1992;51:516-25.

14 Sherman SL, Petersen MB, Freeman SB, et al. Non-disjunction of chromosome 21 in maternal meiosis I: evidence for a maternal age-dependent mechanism involving reduced recombination. Hum Molec Genet 1994;3:1529-35.

15 Stewart GD, Hassold TJ, Berg A, Watkins P, Tanzi R, Kurnit DM. Trisomy 21 (Down syndrome): studying nondisjunction and meiotic recombination by using cytonondisjunction and meiotic recombination by using cytogenetic and molecular polymorphisms that span

16 Lorber BJ, Grantham M, Peters J, Willard HF, Hassold TJ. Nondisjunction of chromosome 21: comparisons of cytogenetic and molecular studies of the meiotic stage and parent of origin. Am $\mathcal{F}$ Hum Genet 1992;51:1265-76.

17 Tanzi RE, Watkins PC, Stewart GD, Wexler NS, Gusella $\mathrm{JF}$, Haines $\mathrm{K}$. A genetic linkage map of human chromosome 21: analysis of recombination as a function of sex and age. Am $\mathcal{F}$ Hum Genet 1992;50:551-8.

18 MacDonald $M$, Hassold $T$, Harvey J, Wang LH, Morton NE, Jacobs P. The origin of $47, X X Y$ and $47, X X X$ aneuploidy: heterogeneous mechanisms and role of aberrant recombination. Hum Molec Genet 1994;3:1365-71.

19 Sambrook J, Fritsch EF, Maniatis T. Molecular cloning: laboratory manual. 2nd ed. New York: Cold Spring Harbor Laboratory Press, 1989.

20 McInnis MG, Chakravarti A, Blaschak J, et al. A linkage map of human chromosome 21: 43 PCR markers a average intervals of $2.5 \mathrm{cM}$. Genomics 1993;16:562-71.

21 Warren AC, Petersen MB, Hul WV, et al. D21S215 is (GT) polymorphic marker close to centromeric alphoid sequences on chromosome 21 . Genomics 1992;13:1365-7.

22 Schlessinger D, Mandel J-L, Monaco AP, Nelson DL, Willard HF. Report of the fourth international workshop on human X chromosome mapping 1993. Cytogenet Cell Genet 1993;64:147-94.

23 Browne DL, Luty JA, Litt M. Dinucleotide repeat polymorphism at the DXS538 locus. Nucleic Acids Res 1991; morphism

24 Borgaonkar DS. Chromosomal variation in man: a catalog of chromosomal variants and anomalies, 5th ed. New York: chromosomal variants and and
Alan R Liss, 1989:689-90.

25 Zaragoza MV, Jacobs PA, James RS, Rogan P, Sherman S, Hassold T. Nondisjunction of human acrocentric chromosomes: studies of 432 trisomic fetuses and liveborns. Hum Genet 1994;94:411-17.

26 Hassold T, Pettay D, May K, Robinson A. Analysis of nondisjunction in sex chromosome tetrasomy and pentasomy. Hum Genet 1990;85:648-50.

27 David D, Marques RA, Carreiro MH, Moreira I, Boavida MG. Parental origin of extra chromosomes in persons with X chromosome tetrasomy. $\mathcal{F}$ Med Genet 1992;29: 595-6.

28 Leal CA, Belmont JW, Nachtman R, Cantu JM, Medina C. Parental origin of the extra chromosomes in polysomy X. Hum Genet 1994;94:423-6.

29 Martini G, Carillo G, Catizone F, Notarangelo A, Mingarell $R$, Dallapiccola B. On the parental origin of the X's in a prenatally diagnosed 49,XXXXX syndrome. Prenat Diagn 1993;13:763-6.

30 Lejeune J. Autosomal disorders. Pediatrics 1963;32:326-37. 1 Koshland D. Mitosis: back to the basics. Cell 1994;77 951-4.

32 Carpenter ATC. Chiasma function. Cell 1994;77:959-72.

33 Hawley RS, Frazier JA, Rasooly R. Commentary. Separation anxiety: the etiology of nondisjunction in flies and people. Hum Molec Genet 1994;3:1529-35. 